\title{
Electron and Exciton Quasi-Stationary $s$-States in Open Spherical Quantum Dots
}

\author{
M.V. TKaCh*, Ju.O. Seti And O.M. Voitsekhivska \\ Chernivtsi National University, Kotsiybynsky Str. 2, Chernivtsi, 58012, Ukraine
}

(Received December 10, 2011; in final form February 10, 2012)

\begin{abstract}
The theoretical calculation of spectral parameters of electron and exciton quasi-stationary $s$-states in open spherical quantum dot is performed within the effective mass approximation and rectangular potentials model. The conceptions of probability distribution functions (over quasi-momentum or energy) of electron location inside of quantum dot and their spectral characteristics: generalized resonance energies and widths are introduced. It is shown that the generalized resonance energies and widths, obtained within the distribution functions, satisfy the Heisenberg uncertainty principle for the barrier widths varying from zero to infinity. At the same time, the ordinary resonance energies and widths defined as complex poles of scattering $S$-matrix, do not satisfy it for the small barrier widths and, therefore, are correct only for the open quantum dots with rather wide potential barriers.
\end{abstract}

PACS: 71.15.Dx, 73.21.La, 73.22.Dj, 73.90.+f

\section{Introduction}

The modern experimental abilities of infrared range cascade lasers, resonance tunnel diodes and separable quantum dots production and wide perspectives of their utilization in microbiology and medicine [1-4] constantly stimulate the interest of theoretical investigations of open nanostructures. The explaining of all physical phenomena in them is connected with the description of electron and exciton quasi-stationary spectra and interaction of these quasi-particles with classic and quantized fields.

The electron spectrum in open quantum films, wires and dots is studied using different theoretical methods [5-11]. In the framework of the effective mass approximation and rectangular potential barriers model, the quasi-stationary electron spectrum in open spherical quantum dot (QD) is usually studied within the scattering $S$-matrix method $[7,8,11]$ because it allows the exact solution of the Schrödinger equation. The complex poles of $S$-matrix define the resonance energies (REs) and resonance widths (RWs) of electron in open spherical QD with wide barriers rather well [11].

Nevertheless, it is already established that the quasi-stationary electron (exciton) spectrum in open spherical QD with thin and super thin barriers, the most perspective for the practical utilization, cannot be defined by the complex poles of $S$-matrix [11].

In this paper, the new characteristics of electron and exciton states in open spherical QDs: the generalized

* corresponding author; e-mail: ktf@chnu.edu.ua resonance energies (GREs) and generalized resonance widths (GRWs) valid at arbitrary potential barrier width are introduced and studied. It is also proven that the universal characteristic of electron or exciton quasi-stationary states (QSSs) in open spherical QD is the probability distribution function (over quasi-momentum or energy) of quasi- particle located inside of QD. The dependences of electron and exciton GREs and GRWs on the barrier width is studied for InAs/GaAs/InAs nanostructure.

\section{2. $S$-matrix and probability distribution functions of quasi-particles located inside of open spherical QD}

The open spherical QD (Fig. 1) consisting of semiconductor core (0) inside of the shell (1) embedded into the outer medium (2) is under study. The radius of core-well is $r_{0}$ and thickness of barrier-shell is $\Delta$. In spherical coordinate system with the beginning in $\mathrm{QD}$ center, the electron or hole effective masses and potential energies are fixed by the expressions

$$
\begin{aligned}
& m(r)=\left\{\begin{array}{l}
m_{0}, \\
m_{1},
\end{array}\right. \\
& U(r)= \begin{cases}0, & 0 \leq r \leq r_{0}, \quad r_{0}+\Delta \leq r<\infty, \\
U, & r_{0} \leq r \leq r_{1}=r_{0}+\Delta .\end{cases}
\end{aligned}
$$

Using the Hamiltonian of the system 


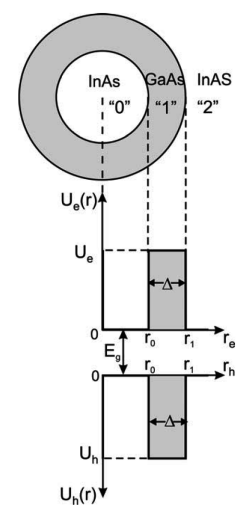

Fig. 1. Geometrical and potential energy schemes of open spherical QD.

$$
H=-\frac{\hbar^{2}}{2} \nabla \frac{1}{m(r)} \nabla+U(\boldsymbol{r}),
$$

we solve the stationary Schrödinger equation exactly and obtain the complete set of wave functions

$$
\Psi_{\ell m k}(r, \theta, \varphi)=R_{\ell k}(r) Y_{\ell m}(\theta, \varphi) .
$$

Here, $Y_{\ell m}(\theta, \varphi)$ - the spherical functions $(\ell=$ $0,1,2, \ldots ; m=0, \pm 1, \pm 2, \ldots)$ and the radial ones $R_{\ell k}(r)$ are taken as linear combinations of the Hankel functions

$$
R_{\ell k}=\left\{\begin{array}{l}
R_{\ell}^{(0)}(k r)=a_{\ell}^{(0)}\left[h_{\ell}^{-}(k r)+h_{\ell}^{+}(k r)\right], \\
0 \leq r \leq r_{0}, \\
R_{\ell}^{(1)}(k r)=a_{\ell}^{(1)}\left[h_{\ell}^{-}(\mathrm{i} \chi r)+S_{\ell}^{(1)} h_{\ell}^{+}(\mathrm{i} \chi r)\right], \\
r_{0} \leq r \leq r_{1}=r_{0}+\Delta, \\
R_{\ell}^{(2)}(k r)=a_{\ell}^{(2)}\left[h_{\ell}^{-}(k r)+S_{\ell}(k) h_{\ell}^{+}(k r)\right], \\
r_{0}+\Delta \leq r<\infty,
\end{array}\right.
$$

where

$$
\begin{aligned}
& k=\hbar^{-1} \sqrt{2 m_{0} E}, \quad \chi=\sqrt{\left(k_{0}^{2}-k^{2}\right) m_{1} / m_{0}}, \\
& k_{0}=\hbar^{-1} \sqrt{2 m_{0} U} .
\end{aligned}
$$

The continuity conditions of radial wave functions and their densities of currents at all nanostructure interfaces together with the normalizing one, define all unknown coefficients and scattering $S_{\ell}(k)$-matrix [7, 8, 11].

Further, avoiding the sophisticated expressions, we are going to observe only the $s$-states $(\ell=0)$. The exact analytical expression for $S_{0}(k) \equiv S(k)$ matrix in under barrier region of energy spectrum $\left(E \leq U, k \leq k_{0}\right)$ is conveniently written in the form

$$
S(k)=\mathrm{e}^{-2 \mathrm{i} k r_{1}} \frac{1+\mathrm{i} Z(k)}{1-\mathrm{i} Z(k)},
$$

where

$$
Z(k)=\frac{k r_{1}}{1-\frac{m_{0}}{m_{1}}\left[1-\chi r_{1} \frac{1+\xi(k) \exp (-2 \chi \Delta)}{1-\xi(k) \exp (-2 \chi \Delta)}\right]},
$$

$$
\xi(k)=\frac{m_{1} k r_{0} \cot \left(k r_{0}\right)-m_{0} \chi r_{0}+m_{0}-m_{1}}{m_{1} k r_{0} \cot \left(k r_{0}\right)+m_{0} \chi r_{0}+m_{0}-m_{1}} .
$$

$S(k)$-matrix (6), of course, coincides to the one obtained in Refs. $[7,8,11]$ in other analytical form, but its expression through the real $Z(k)$ function has the advantages which would be clear further. Especially, the expression (6) is valid for $k>k_{0}(E>U)$ at the condition $\chi \rightarrow \mathrm{i} \chi$ in $Z(k)$ function.

Now, we introduce the probability distribution functions $W(k)$ or $W(E)$ (over quasi- momentum or energy) of quasi-particle located inside of open spherical QD (in the sphere of $r_{1}=r_{0}+\Delta$ radius)

$$
\begin{aligned}
& W(k)=\frac{1}{r_{1}} \int_{0}^{r_{1}}|X(k r)|^{2} \mathrm{~d} r, \\
& W(E)=\frac{1}{r_{1}} \int_{0}^{r_{1}}\left|X_{E}(r)\right|^{2} \mathrm{~d} r,
\end{aligned}
$$

where

$$
X(k r)=r R(k r), \quad X_{E}=r R_{E}(r) .
$$

The calculation of these functions is analytically performed exactly. Really, using Schrödinger Eq. (2) for the two close energy values ( $E$ and $E_{1}$ ) we obtain

$$
\begin{aligned}
& W(E)=\frac{1}{r_{1}} \frac{\hbar^{2}}{2 m_{0}} \lim _{E_{1} \rightarrow E} \frac{1}{E_{1}-E}\left[X_{\mathrm{E}_{1}}(r) X_{E}^{\prime}(r)\right. \\
& \left.-X_{\mathrm{E}_{1}}^{\prime}(r) X_{E}(r)\right]\left.\right|_{r=r_{1}} .
\end{aligned}
$$

According to the general theory $[12,13]$, for $r \geq r_{1}$ where $U(r)=0, X_{E}^{(2)}(r)$ function is written as

$$
X_{E}^{(2)}(r)=\sqrt{\frac{2}{\pi}} \sin (k r+\delta),
$$

where the phase $(\delta)$ is related to the $S$-matrix through the expression

$$
S(k)=\mathrm{e}^{2 \mathrm{i} \delta(k)},
$$

after some transformations it is obtained

$$
W(k)=\frac{k}{\pi r_{1}}\left[1+Z^{2}(k)\right]^{-1} \frac{\mathrm{d}}{\mathrm{d} k}\left(\frac{Z(k)}{k}\right) .
$$

Taking into account the analytical form of $Z(k)(7)$, we get the exact and convenient for calculations expression for the probability distribution function at $k \leq k_{0}$ :

$$
\begin{aligned}
& W(k)=\frac{k r_{1}}{\pi}\left\{\sqrt{\frac{m_{0}}{m_{1}}} \frac{k}{\chi}\left[\xi^{2}+\exp (-4 \chi \Delta)\right]\right. \\
& \left.+2 \exp (-2 \chi \Delta)\left(\chi \frac{m_{0}}{m_{1}} \xi^{\prime}-\sqrt{\frac{m_{0}}{m_{1}}} 2 \xi \Delta\right)\right\} \\
& /\left([ 1 + Z ( k ) ^ { 2 } ] \left\{\chi r_{1} \frac{m_{0}}{m_{1}}[\xi+\exp (-2 \chi \Delta)]\right.\right. \\
& \left.\left.+\frac{m_{1}-m_{0}}{m_{1}}[\xi-\exp (-2 \chi \Delta)]\right\}\right),
\end{aligned}
$$

where

$$
\xi^{\prime}=2 \frac{m_{0}}{m_{1}} r_{0}\left\{k \chi r_{0}^{2}\left[1+\cot ^{2}\left(k r_{0}\right)\right]\right.
$$




$$
\begin{aligned}
& \left.-\left(1+\sqrt{\frac{m_{1}}{m_{0}}} \frac{k^{2}}{\chi^{2}}\right) \chi r_{0} \cot \left(k r_{0}\right)+\frac{m_{1}-m_{0}}{m_{1}} \sqrt{\frac{m_{1}}{m_{0}}} \frac{k}{\chi}\right\} \\
& /\left\{\left[k r_{0} \cot \left(k r_{0}\right)-\frac{m_{0}}{m_{1}} \chi r_{0}-\frac{m_{1}-m_{0}}{m_{1}}\right]^{2}\right\} \cdot
\end{aligned}
$$

Expression (15) for the distribution function $W(k)$ (and equivalent to it $W(E)$ ) stays valid also for $k \geq k_{0}$ $(E \geq U)$ when $\chi \rightarrow \mathrm{i} \chi$. Further, it is proven that just $W(E)$ distribution function allows introducing the GRE and GRW conceptions which are true independently of the width of the open spherical potential barrier.

The Hamiltonian of exciton has the form

$$
H_{\mathrm{ex}}=E_{\mathrm{g}}+H_{e}\left(r_{e}\right)+H_{h}\left(r_{h}\right)-\frac{e^{2}}{\varepsilon\left|r_{e}-r_{h}\right|} .
$$

Here, $E_{\mathrm{g}}$ - band gap energy, $H_{e}\left(r_{e}\right), H_{h}\left(r_{h}\right)$ - the electron and hole Hamiltonians, expression (2) and $\varepsilon-$ dielectric constant of " 0 " and " 2 " media where the quasi-particles are mainly located.

The Schrödinger equation with Hamiltonian (17) cannot be solved exactly. Thus, the approximated method is used. When the sum of uncoupling electron and hole resonance energies in the respective exciton QSS is much bigger than the energy of their interaction in these states, it can be assumed that the probability distribution function over quasimomentum for the exciton located in open spherical QD is given by the expression

$$
\begin{aligned}
& W\left(k_{e}, k_{h}\right)=W\left(k_{e}\right) W\left(k_{h}\right) \\
& \quad=\frac{k_{e} k_{h}}{\pi^{2} r_{1}^{2}} \frac{\mathrm{d}\left[k_{e}^{-1} Z_{e}\left(k_{e}\right)\right] / \mathrm{d} k_{e}}{\left[1+Z_{e}^{2}\left(k_{e}\right)\right]} \frac{\mathrm{d}\left[k_{h}^{-1} Z_{h}\left(k_{h}\right)\right] / \mathrm{d} k_{h}}{\left[1+Z_{h}^{2}\left(k_{h}\right)\right]} .
\end{aligned}
$$

Using (5) and (18), we obtain the probability distribution function over the energy for the exciton located in open spherical QD

$$
W\left(E_{e}, E_{h}\right)=W\left(E_{e}\right) W\left(E_{h}\right),
$$

fixing the exciton GREs and GRWs in spherically-symmetric states $(\ell=0)$.

\section{Electron and exciton quasi-stationary $s$-states in open spherical QD}

The calculation of the probability distribution functions of electron and exciton located in open spherical QD is performed according to the developed theory for InAs/GaAs/InAs nanostructure with physical parameters presented in Table.

TABLE

Physical parameters of InAs/GaAs/InAs nanostructure.

\begin{tabular}{c|c|c|c|c|c|c}
\hline \hline & $m_{e}$ & $m_{h}$ & $a[\AA]$ & $\varepsilon_{\infty}$ & $\varepsilon_{0}$ & $E_{\mathrm{g}}[\mathrm{eV}]$ \\
\hline InAs & 0.022 & 0.41 & 6.058 & 15.1 & 16.3 & 0.35 \\
GaAs & 0.067 & 0.5 & 5.653 & 10.9 & 12.9 & 1.52
\end{tabular}

Let us, first of all, analyze the main properties of $W(k)$ and $W(E)$ functions for the electron. They would give the opportunity to introduce the conceptions of GREs and GRWs of QSSs in such a way that the latter would be valid for the whole infinite range of barrier widths ( $\Delta$ is given in the units of InAs lattice constant $a$ ) and at $\chi \Delta>1$ would coincide to the usual REs and RWs obtained from the complex poles of $S$-matrix according to Refs. [12, 13].

The electron distribution functions $W\left(K=k r_{0}\right)$ and $W(E)$ are shown in Fig. 2. It is clear that their properties are different for different barrier widths: small $\left(0 \leq \Delta \ll r_{0}\right)$, relative $\left(\Delta \leq r_{0}\right)$; and big $\left(\Delta>r_{0}\right)$. Let us observe $W(K)$ and $W(E)$ peculiarities for the small $\Delta$ magnitudes (Fig. 2). When the potential barrier is absent $(\Delta=0)$, from the expression for probability distribution function

$$
\begin{aligned}
& \left.W(K)\right|_{\Delta=0}=\frac{1}{\pi}\left(1-j_{0}(K)\right)=\frac{1}{\pi}\left[1-\frac{\sin (2 K)}{2 K}\right], \\
& K=k r_{0},
\end{aligned}
$$

and Fig. 2 one can see that $W(K)$ and $W(E)$ functions perform the quasi-periodical oscillations respectively the average value

$$
\left.\bar{W}\right|_{\Delta=0}=\lim _{A \rightarrow \infty} \frac{1}{A} \int_{0}^{\infty} W(K) \mathrm{d} K=\frac{1}{\pi}
$$

consistently taking minimum $\left(W_{n}^{<}=\frac{2}{\pi} \sin ^{2} k_{n}^{<} r_{0}\right)$ and maximum $\left(W_{n}^{>}=\frac{2}{\pi} \sin ^{2} k_{n}^{>} r_{0}\right)$ values, $(n=$ $0,1,2, \ldots, \infty)$ at $k_{n}^{<}=r_{0}^{-1} K_{2 n}, k_{n}^{>}=r_{0}^{-1} K_{2 n+1}$, where $K_{2 n}$ and $K_{2 n+1}$ are the even and odd roots of the equation

$$
\left.W^{\prime}(K)\right|_{\Delta=0}=0, \quad \text { or } \quad K \cot (K)=1 .
$$

The oscillations of $W(K)$ and $W(E)$ functions in $K$ - or $E$-scales, respectively, create the continuous sequence of peaks $\left(n_{e}=1,2,3, \ldots \infty\right)$, each characterized by its maximum and width. As far as it is true for any width, it is reasonable to introduce two main spectral characteristics of $n$-th electron peak: GRE: $E_{n_{e}}^{w}=\hbar^{2}\left(k_{n_{e}}^{>}\right)^{2} /\left(2 m_{0}\right)$, corresponding to the maximum of $W_{n_{e}}\left(K_{n_{e}}^{>}\right)$, and GRW: $\Gamma_{n_{e}}^{w}=E_{n_{e}}^{(+)}-E_{n_{e}}^{(-)}$, where $E_{n_{e}}^{( \pm)}$energies are the roots of the equation, defined by the natural condition (Fig. 2):

$$
2 W(E)=W\left(K_{n_{e}}^{>}\right)+W\left(K_{n_{e}}^{<}\right) .
$$

From Fig. 2 one can see that for increasing $\Delta$, the heights of all under barrier peaks are growing in the vicinity of resonances due to the decrease of $W$ in intervals between the resonances. Since, at the increasing $\Delta$ these peaks have at first the quasi-Lorentz shape and then consistently transform into $\delta$-like functions with maxima at $K \sim K_{n_{e}}$. At limit case $\Delta \rightarrow \infty$, GRWs of under barrier QSSs tend to zero $\left(\Gamma_{n_{e}}^{w} \rightarrow 0\right)$ and their GREs $\left(E_{n_{e}}^{w}\right)$ coincide to the stationary electron energy spectrum $\left(E_{n_{e}}^{c}\right)$ in closed spherical QD, as it must be according to the physical considerations.

Analyzing now the spectral parameters: GREs and GRWs of the electron QSSs in open spherical QD, we must note that they satisfy Heisenberg uncertainty prin- 


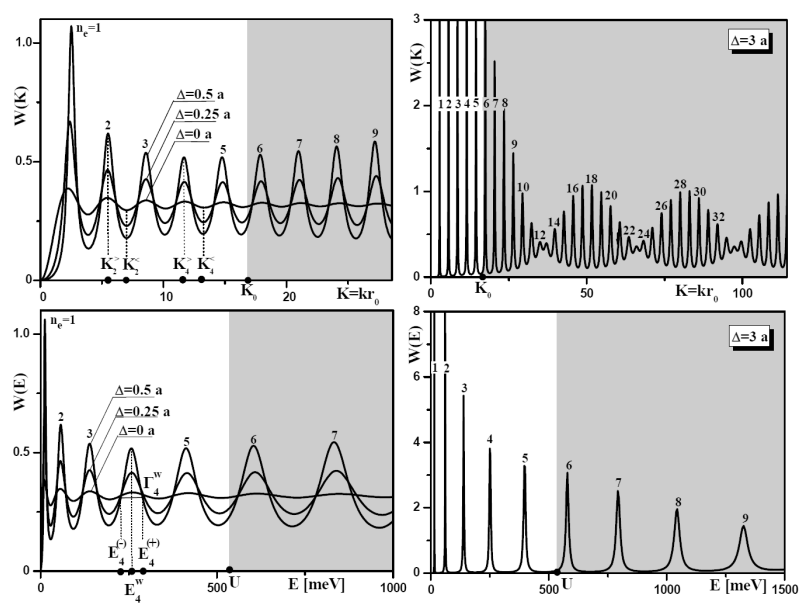

Fig. 2. Evolution of electron probability distribution functions $\left(W\left(K=k r_{0}\right)\right.$ and $\left.W(E)\right)$ at the small barrier width of open spherical QD and $r_{0}=50 a$.

ciple at the whole range of barrier widths $(0 \leq \Delta<\infty)$. Also, for the big barrier widths they must be equal to the REs and RWs defined from the complex poles of scattering $S$-matrix.
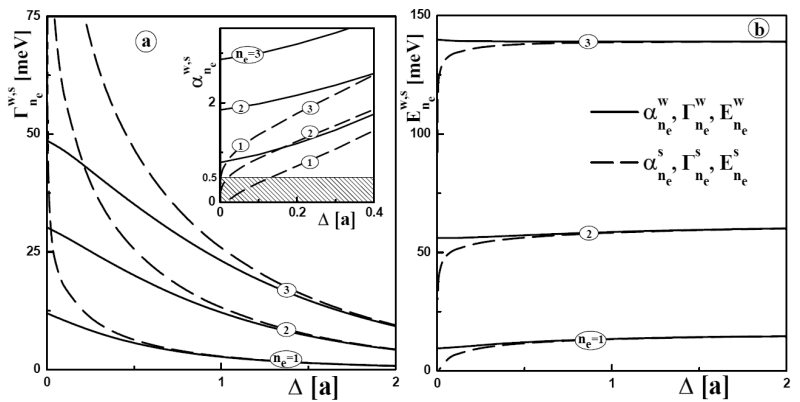

Fig. 3. Dependences of electron spectral parameters $\Gamma_{n_{e}}^{w, s}(\mathrm{a})$ and $E_{n_{e}}^{w, s}(\mathrm{~b})$ on the barrier width $\Delta$.

In Fig. 3, where $E_{n_{e}}^{w}, \Gamma_{n_{e}}^{w}$ and $E_{n_{e}}^{s}, \Gamma_{n_{e}}^{s}$ dependences on $\Delta$ are shown, one can see that GREs and GRWs satisfy all abovementioned demands. From Fig. 3a,b it is clear that for the under barrier energies $(E<U)$ for the barrier of one monoshell $(a)$ order or bigger, the QSSs spectral parameters $\left(E_{n_{e}}^{w}, E_{n_{e}}^{s}\right.$ and $\left.\Gamma_{n_{e}}^{w}, \Gamma_{n_{e}}^{s}\right)$, defined within $W$ function and $S$-matrix are better coinciding between each other, the bigger is the width $\Delta$. When the barrier width is smaller than $a$ and tends to zero, the REs $\left(E_{n_{e}}^{s}\right)$ also tend to zero and RWs $\left(\Gamma_{n_{e}}^{s}\right)$ increase tending to infinity. It causes that $\alpha_{n_{e}}^{s}(\Delta)=E_{n_{e}}^{s} / \Gamma_{n_{e}}^{s}$ (dash curves at inset in Fig. 3a) at small $\Delta$ become smaller than $1 / 2$, contradicting the Heisenberg principle $\left(E_{n_{e}} t_{n_{e}}=\hbar E_{n_{e}} / \Gamma_{n_{e}}=\hbar \alpha_{n_{e}}^{s} \geq \hbar / 2\right)$. As far as GREs $\left(E_{n_{e}}^{w}\right)$ and GRWs $\left(\Gamma_{n_{e}}^{w}\right)$ are concerned, at $\Delta$ tending to zero, the both parameters tend to the finite magnitudes always satisfying Heisenberg principle (solid curves at the inset). Finally, let us note that at $\Delta \rightarrow \infty$
$\Gamma_{n_{e}}^{w} \rightarrow \Gamma_{n_{e}}^{s} \rightarrow 0$ and $E_{n_{e}}^{w} \rightarrow E_{n_{e}}^{s} \rightarrow E_{n_{e}}^{c}$, where $E_{n_{e}}^{c}-$ energies of bound stationary states of electron in closed spherical QD.

The GREs, REs and GRWs, RWs of exciton QSSs are calculated within the approximated method, using the following considerations. When the electron-hole interaction is neglected, the energies and widths of exciton QSS $s$-states are defined, depending on the method, by the formulae

$$
\begin{aligned}
& E_{n_{e} n_{h}}^{(0) w, s}=E_{\mathrm{g}}+E_{n_{e}}^{w, s}+E_{n_{h}}^{w, s} ; \\
& \Gamma_{n_{e} n_{h}}^{(0) w, s}=\Gamma_{n_{e}}^{w, s}+\Gamma_{n_{h}}^{w, s},
\end{aligned}
$$

where $E_{n_{e} n_{h}}^{w}, \Gamma_{n_{e} n_{h}}^{w}$ - the GREs and GRWs of electron (e) and hole (h) QSSs, obtained before within the probability distribution function and $E_{n_{e} n_{h}}^{s}, \Gamma_{n_{e} n_{h}}^{s}$ - the REs and RWs of the same states, obtained as the complex poles of $S$-matrix.

The Coulomb potential energy of electron-hole interaction does not create the additional potential barrier for the transition of both quasi-particles from QD. Thus, we assume that it is only renormalizing the energy of exciton QSSs without changing their widths in the first approximation. The renormalized energies of QSSs $\left|n_{e} n_{h}\right\rangle$ in open nanostructure cannot be calculated using the wave functions normalized at $\delta$-function. Therefore, the calculation of electron-hole binding energy $\left(\Delta E_{n_{e} n_{h}}\right)$ is performed as in Ref. [14]. Instead of the single open spherical QD we observe the equivalent two-well closed spherical QD with so big width of the outer shell-well that the energies and widths of "former" resonance states with good exactness coincide to the respective REs and RWs in open QD. Herein, we use the perturbation theory and in the first approximation the corrections to the exciton REs are written as

$$
\begin{aligned}
& \Delta E_{n_{e} n_{h}}=-\frac{e^{2}}{\varepsilon} \int_{0}^{\infty} \mathrm{d} r_{e} \int_{0}^{\infty} \mathrm{d} r_{h}\left|R_{n_{e}}\left(r_{e}\right)\right|^{2}\left|R_{n_{h}}\left(r_{h}\right)\right|^{2} \\
& \quad \times r_{e}^{2} r_{h}^{2} \begin{cases}r_{e}^{-1}, & r_{e} \geq r_{h}, \\
r_{h}^{-1}, & r_{h} \geq r_{e},\end{cases}
\end{aligned}
$$

where $R_{n_{e}}\left(r_{e}\right), R_{n_{h}}\left(r_{h}\right)$ - electron (e) and hole (h) wave functions in the states $n_{e}$ and $n_{h}$ of two-well closed spherical QD, approximating open spherical QD [14].

Thus, the spectral characteristics: energies $\left(E_{n_{e} n_{h}}^{w, s}\right)$ and widths $\left(\Gamma_{n_{e} n_{h}}^{w, s}\right)$ are fixed by the expressions

$$
\begin{aligned}
& E_{n_{e} n_{h}}^{w, s}=E_{\mathrm{g}}+\Delta E_{n_{e} n_{h}}+E_{n_{e}}^{w, s}+E_{n_{h}}^{w, s} ; \\
& \Gamma_{n_{e} n_{h}}^{w, s}=\Gamma_{n_{e}}^{w, s}+\Gamma_{n_{h}}^{w, s} .
\end{aligned}
$$

The numeric calculations of exciton REs $\left(E_{n_{e} n_{h}}^{s}\right)$, RWs $\left(\Gamma_{n_{e} n_{h}}^{s}\right)$ and GREs $\left(E_{n_{e} n_{h}}^{w}\right)$, GRWs $\left(\Gamma_{n_{e} n_{h}}^{w}\right)$ are performed for open spherical QD InAs/GaAs/InAs. Herein, $E_{n_{e}}^{s}$, $E_{n_{h}}^{s}$ and $\Gamma_{n_{e}}^{s}, \Gamma_{n_{h}}^{s}$ are fixed by the complex poles of the respective $S_{e^{-}}$and $S_{h^{-}}$matrixes. The generalized energies, fixed by the formulae: $E_{n_{e}}^{w}=\hbar^{2}\left(k_{n_{e}}^{>}\right)^{2} / 2 m_{e}$, $E_{n_{h}}^{w}=\hbar^{2}\left(k_{n_{h}}^{>}\right)^{2} / 2 m_{h}$ and widths: $\Gamma_{n_{e}}^{w}=E_{n_{e}}^{(+)}-E_{n_{e}}^{(-)}$, $\Gamma_{n_{h}}^{w}=E_{n_{h}}^{(+)}-E_{n_{h}}^{(-)}$are defined as the corresponding spectral parameters of probability distribution function 
for exciton in QD (Fig. 4, without accounting of the band gap energy $E_{\mathrm{g}}$ ). In Fig. 4a the spatial shape of probability distribution function $W\left(k_{e}, k_{h}\right)$ in $k$-space is presented. In Fig. $4 \mathrm{~b}$ the first peak of $W\left(E_{e}, E_{h}\right)$ distribution function together with the respective terms of generalized energies $\left(E_{e 1}^{w}, E_{h 1}^{w}\right)$ and widths $\left(\Gamma_{e 1}^{w}, \Gamma_{h 1}^{w}\right)$ are shown.
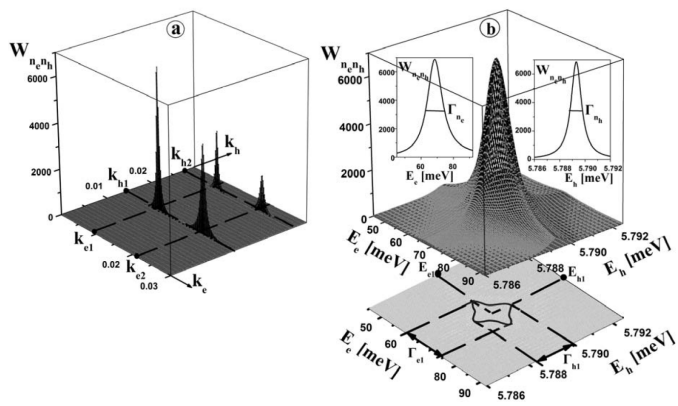

Fig. 4. Probability distribution functions $W\left(k_{e}, k_{h}\right)$ (a), $W\left(E_{e}, E_{h}\right)$ (b) and spectral parameters of electron and hole quasi-stationary states.
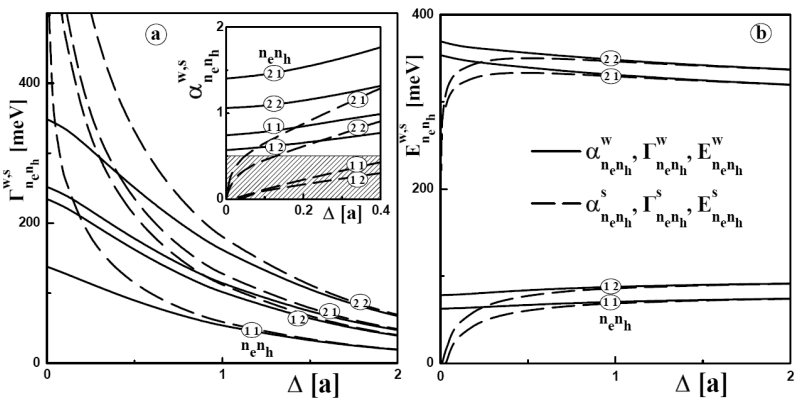

Fig. 5. Dependences of exciton spectral parameters $\Gamma_{n_{e} n_{h}}^{w, s}$ (a) and $E_{n_{e} n_{h}}^{w, s}$ (b) on the barrier width $\Delta$.

The typical dependences of generalized resonance $(\mathrm{w})$ and resonance (s) spectral parameters of several exciton QSSs on the barrier width $(\Delta)$ are given in Fig. 5 (without accounting of the band gap energy $E_{\mathrm{g}}$ ). The figure proves that the exciton spectral parameters (the same for the electron and hole), determined by both methods, coincide well at $\Delta \geq 2 a \geq 1 \mathrm{~nm}$ and strongly differ for the small barrier widths. The GREs of exciton QSSs weakly depend on barrier thickness and GRWs increase, approaching some finite magnitudes for the smaller barrier widths. For the rather big widths, the REs and RWs, obtained from the complex poles of $S$-matrix give the true magnitudes while at small $\Delta$ these magnitudes are not correct because they contradict the Heisenberg uncertainty principle.

\section{Conclusions}

The spectral parameters (REs and RWs) of spherically symmetric electron, hole and exciton QSSs in open spherical QD calculated within the method of complex $S$-matrix poles are true in the wide range of barrier widths $(\Delta)$ except the small ones $\left(\Delta \ll r_{0}\right)$, where these magnitudes contradict the Heisenberg uncertainty principle.

The probability distribution functions $(W(k), W(E))$ of electron located inside of open spherical QD and their spectral parameters (GREs and GRWs) correctly describe the spherically symmetric QSSs in the whole infinite range of barrier widths satisfying the Heisenberg principle.

The proposed method of electron, hole and exciton GREs and GRWs calculation, after several modifications, can be also used for the multishell open quantum dots, wires and films. Thus, it would be actual for the evaluation of spectral characteristics of QSSs in resonance tunnel devices produced at the base of open nanostructures with thin barriers.

\section{References}

[1] P.H. Siegel, IEEE Trans. Microwave Theory Tech. 52, 2438 (2004).

[2] W.C.W. Chan, S. Nie, Science 281, 2016 (1998).

[3] X. Michalet, F.F. Pihaud, L.A. Bentolila, J.M. Tsay, S. Doose, J.J. Li, G. Sundaresan, A.M. Wu, S.S. Gambhir, S. Weiss, Science 307, 538 (2005).

[4] X. Gao, Y. Cui, R.M. Levenson, L.W.K. Chung, S. Nie, Nature Biotechnol. 22, 969 (2004).

[5] Y.X. Liu, D.Z.-Y. Ting, T.C. McGill, Phys. Rev. B 54, 5675 (1996).

[6] M. Tkach, V. Holovatsky, O. Voitsekhivska, Physica E 11, 17 (2001).

[7] N.V. Tkach, Ju.A. Seti, Semiconductors 40, 1083 (2006).

[8] N.V. Tkach, Yu.A. Seti, G.G. Zegrya, Technical Phys. Lett. 33, 35 (2007).

[9] S.J. Lee, H.C. Jeon, T.W. Kang, S. Souma, Physica E 40, 2198 (2008).

[10] N.V. Tkach, Yu.A. Seti, Low Temp. Phys. 35, 556 (2009).

[11] N.V. Tkach, Ju.A. Seti, Semiconductors 43, 340 (2009).

[12] A I. Baz', Ya.B. Zel'dovich, A.M. Perelomov, Scattering, Reactions and Decays in Nonrelativistic Quantum Mechanics, 2nd ed., Nauka, Moscow 1971, p. 463.

[13] R.G. Newton, Scattering Theory of Waves and Particles, Springer, New York 2002.

[14] M. Tkach, Ju. Seti, Condens. Matter. Phys. 10, 23 (2007). 